\title{
Virtual Reality Implementation of Robot Navigation and Devices Control in Industries
}

\author{
K. Kalaivani, S. Nagarajan, T. Kalaiselvi, B. Chinthamani, J. Johnsi
}

\begin{abstract}
In industrial environment the controlling of large equipment is a difficult task as it involves lot of wiring and connections which is strenuous. Voice recognition based controlling is also not possible due to the deafening sound of the working machines. Moreover the radio frequency signals like infrared signals or a remote cannot be used in the EMI testing areas. This paper presents a suitable method for controlling industrial devices in such an environment using the Virtual Reality concept. It reduces the effort needed for the operation of these devices. This method is a reliable and a simple method. Proposed system minimizes the effort involved in the controlling process and also serves as a method that can be utilized in industrial environments. The basic idea is to project virtual buttons using the virtual reality projector which can be used to operate an industrial device. Once the virtual button pertaining to a particular device.
\end{abstract}

Keywords : Image processing, Navigation, RSSI, Virtual reality

\section{INTRODUCTION}

In the present world, the consumer grade digital cameras and projectors have become less expensive leading to the introduction of many projector-camera based systems. In particular, since ordinary plain walls can be used as the screen, large interactive displays can be used as information boards in public spaces. Touching the screen is intuitive and devices such as smart phones. Our aim is to make large, easy-to-install, economical interactive displays; therefore, we use a front projector and a nearby camera. A hand touch on the screen is detected by the area of the shadow cast by the user's hand.

As the world is fascinated with the Virtual Reality and its applications, many VR applications have been proposed recently. Virtual Reality creates an environment, where the user moves ahead of standard forms of interactions such as the keyboard and mouse, thus taking the human-machine interaction (HMI) to a new level. The experience of a virtual world mimics that of a real world scenario but often without many of its constraints. It allows us to develop a prototype which is tested and the results are fed back to the design team. The advantage of this is that it enables the designers to make alterations to their design but at far less time and cost.

Revised Manuscript Received on 14 September, 2019.

K.Kalaivani, Department of Electronics and Instrumentation Engineering, Easwari Engineering College, Chennai, Tamilnadu, India. (Email: kvani2007@gmail.com)

S.Nagarajan, Department of Electronics and Instrumentation Engineering, Easwari Engineering College, Chennai, Tamilnadu, India.

T.Kalaiselvi, Department of Electronics and Instrumentation Engineering, Easwari Engineering College, Chennai, Tamilnadu, India.

B.Chinthamani, Department of Electronics and Instrumentation Engineering, Easwari Engineering College, Chennai, Tamilnadu, India.

J.Johnsi, Department of Electronics and Instrumentation Engineering, Easwari Engineering College, Chennai, Tamilnadu, India. increasingly popular due to the recent spread of touch-based

In our project, virtual reality makes the control of any devices (extended primarily for industrial purposes) easier and user friendly.

Usually physical buttons are used in almost all appliances and devices for their control. If its functionality or label needs to be changed it involves a tedious and timeconsuming process of modifying the wiring and changing the button type. In noisy environments of industries due to operation of machinery, it is not possible to use voice commands for control. Also, in EMI testing zones the use of $\mathrm{RF}$ and Current signals are prohibited as they can interfere with the process. In order to overcome these setbacks, we have proposed an idea using light for controlling them based on the concept of virtual reality.

Wall mounted buttons are used as physical User Interface (UI) for elevators, room lights and so on. However, changing installation locations of wall mounted buttons is not easy. In addition, changing button labels and types is not easy, too. By changing installation locations, labels and types of wall mounted buttons easily, their User Experience(UX) can be improved. The proposed system uses Virtual Reality(VR) to overcome the shortcomings of wall mounted buttons. We used virtual touch buttons to indicate ON/OFF states, because touch buttons are familiar and fundamental widgets for touch interfaces. They can be projected on a plane wall or any other flat surface. This screen gives a feel of touching the device control unit for controlling the devices. When the screen is touched the selected devices are controlled based upon the touch(either $\mathrm{ON}$ or $\mathrm{OFF}$ ).

The rest of this paper is organized as follows. Section II, deals with the Related works supports this research. Section III presents the detailed architecture of the proposed system architecture and design. Section IV discusses the experimental results related to this work. Finally Section V concludes this paper.

\section{RELATED WORKS}

Virtual reality creates a simulated experience which creates the feel of entering into similar or completely different from real world. In [1], the authors have shown the result by providing a fully automated system to control the electrical devices based on virtual reality concept.

In [2], the authors described the desktop system for robot control providing the gesture based platform for robot control. In [3], the authors described techniques for enabling proficient man-machine interaction in computer augmented multi-surface environments. They focus on explicit

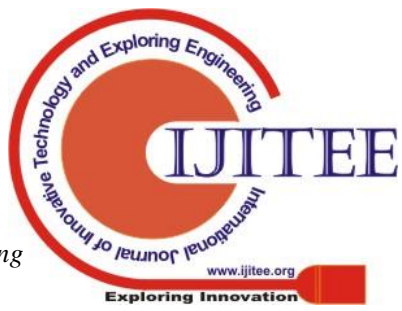


interface, in which the user resolves when and where to communicate with the system.

Besides the traditional ongoing research methodologies for human- machine interfacing, there are several research going on based on virtual reality concepts[4-6].

The existing ideas incorporates voice recognition and hand gesture based controlling. Other methods which exist till date include remote control to operate machines. While the former, cannot be used when the industrial environment which is way too noisy and the gesture control makes use of more computing resources that a mere button touch which is simpler. The latter is not feasible in the industrial testing sectors as radio frequency signals cannot be transmitted as there is hindrance due to electromagnetic signals. The proposed model focus on these issues. On comparison with previous models, it is time-saving and more user-friendly.

\section{PROPOSED SYSTEM ARCHITECTURE AND DESIGN}

This section, presents the architecture and design of Virtual Reality based projector design which is the heart of the system proposed for the control of devices.

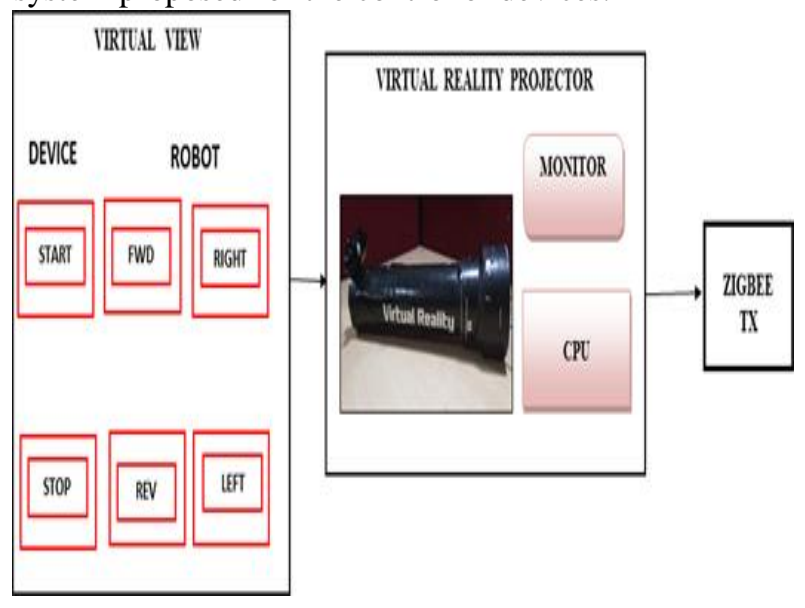

Fig. 1. Block diagram of transmitter

Fig.1 shows the transmitter section of the proposed architecture. The virtual reality projector is used to project the virtual buttons that are used to control the devices in an industry. It has a camera mounted on it to capture the selected virtual button and it is connected to the laptop via USB cable. START and STOP buttons represent the device activation and deactivation. FWD, REV, RIGHT, LEFT buttons indicate the movement of the robot.

The Virtual Reality Projector is connected to a power supply, which projects the virtual buttons of the devices to be controlled. The buttons can be projected on the wall or table or anywhere where the user finds it comfortable which enhances the user experience by making it user-friendly. The user can control the devices of his choice by a mere swipe of his hand. The user should block the virtual button according to his choice to control the device.

The selected button is transmitted to the Visual Basic installed in the laptop via USB cable where image processing takes place, comparing the captured imaged with the original image already stored in Visual Basic. Data signals are transmitted from laptop to Zigbee $2.4 \mathrm{GHz}$ transmitter module that is interfaced with it.

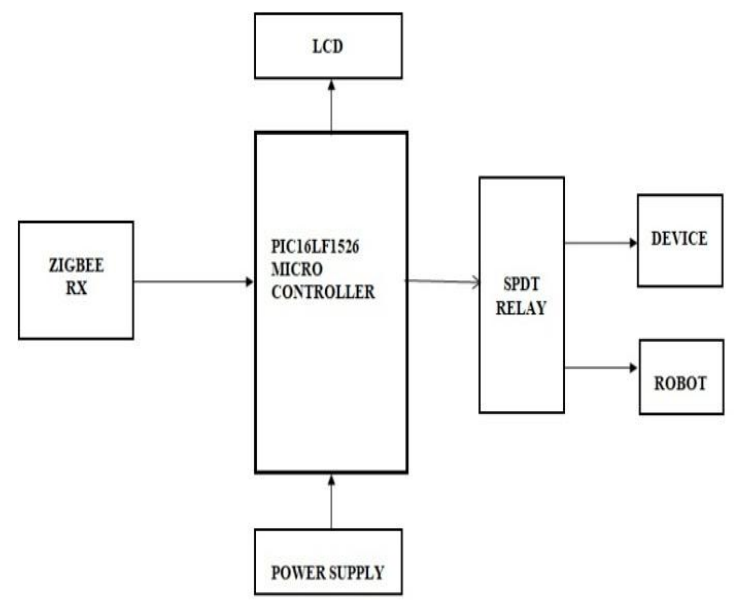

Fig. 2. Block diagram of receiver

Fig.2 shows the receiver section of the proposed architecture. From Zigbee Transmitter, the data signals are sent to the receiver module. The Zigbee 2.4Ghz TI CC2500 receiver module is interfaced with the PIC microcontroller to transmit the processed data from the laptop to the microcontroller. PIC 16LF1526 microcontroller which is an 8 bit microcontroller is used to control the industrial devices.

PIC microcontroller is powered with an adapter which takes $230 \mathrm{~V}$ AC as input and supplies $12 \mathrm{~V} \mathrm{DC}$ as output to power the microcontroller. The PIC Microcontroller sends a command signal (digital signal) to control the SPDT relays that are used to drive the electrical devices and the robot. LCD display shows the operations taking place.

The proposed system has a faster response time in few milliseconds. This model will reduce the power consumption and the increases the ease of control as it can be used at any place any time. This real time model can be put to use in the RF free testing zones in the industries. This system can be used as an alternative for voice recognition as it is not affected by the background noises. So it can be used in industries where there is deafening sound generated due to the machineries.

\section{RESULT AND DISCUSSION}

Fig. 3 presents the hardware set up required to perform the experiment. Hardware setup mainly contains the hardware components of Virtual Reality projector, Zigbee transmitter and receiver pair, 5 SPDT Relay modules and a Robot module. The most important hardware of this prototype is the Virtual Reality(VR) Projector .Virtual reality projector is used to project the virtual buttons that are used to control the devices in an industry. It has a camera mounted on it to capture the selected virtual button and it is connected to the laptop via USB cable. 


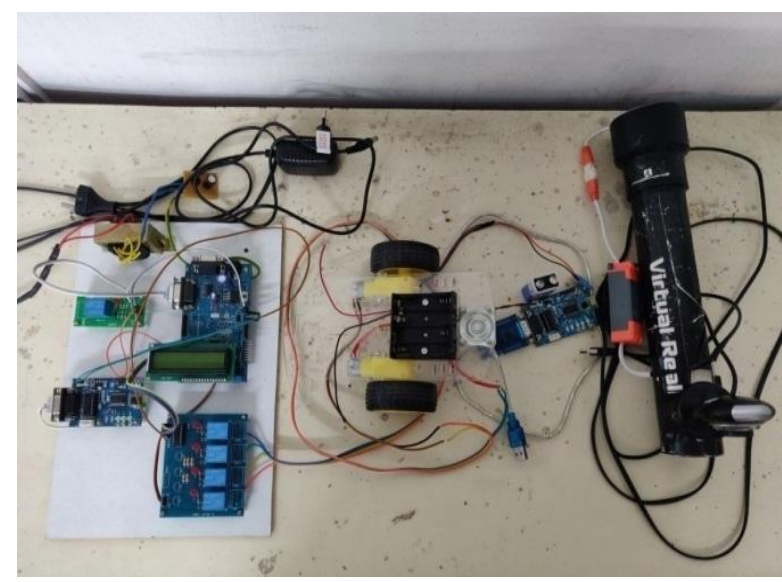

Fig. 3. Hardware setup for the proposed system

Visual Basic is installed in a laptop and used to send a signal to the microcontroller after processing the blocked virtual button by comparing the captured blurred image with the original image. RSSI(Received Signal Strength Indication) module is interfaced with the PIC microcontroller to transmit the processed data. PIC 16LF1526 microcontroller which is an 8 bit microcontroller is used to control the industrial devices.

The model utilises Visual Basic 6.0 and RSSI concept (Received Signal Strength Indication).This former is used for the communication between the virtual reality projector and the laptop ,facilitating the concept of image processing wherein, the standard image is compared to the selected virtual image by comparison of pixel values. The latter is used for communication between the laptop and the PIC microcontroller.

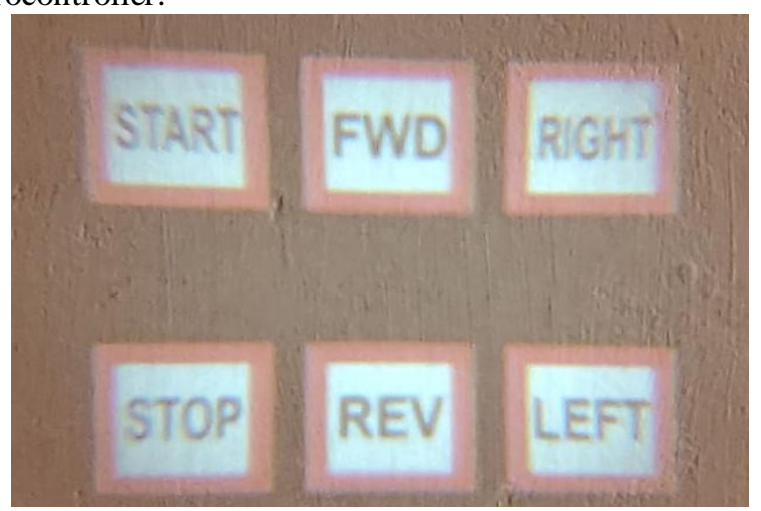

Fig. 4. Projected virtual buttons

Mainly this hardware setup is used to check the functionality of components, sub-assemblies, assemblies and finished product.

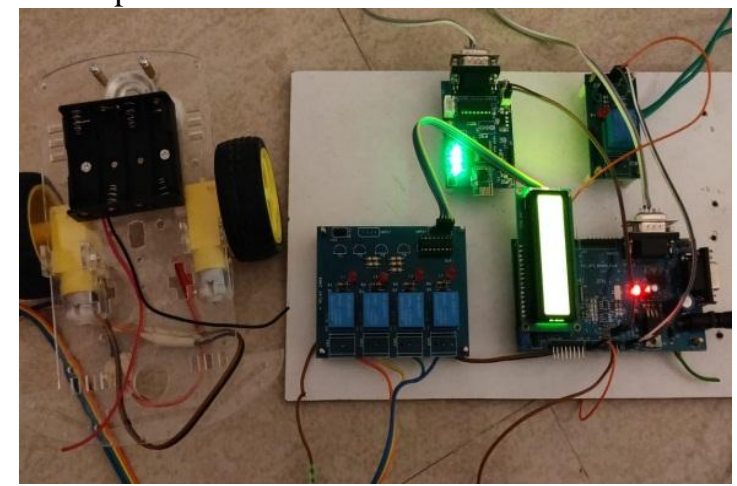

Fig. 5. Connection between Robot and hardware setup
Fig.4 shows the projected virtual buttons using virtual reality projector and Fig.5 depicts connection between Robot and hardware set up for demonstrating Robot navigation. Robot is connected to the microcontroller via SPDT relay which drives the motor for its navigation.

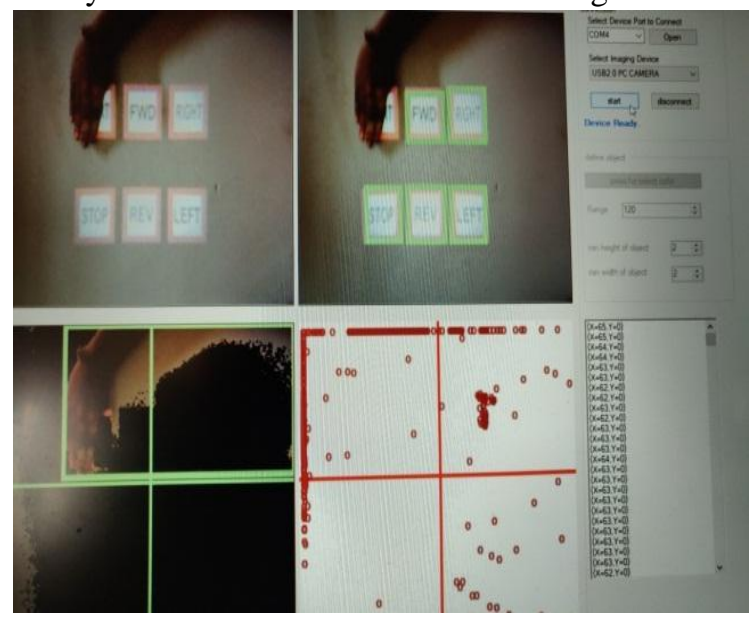

Fig. 6. Image processing in Visual Basic

Fig. 6 shows the results of using image processing techniques in visual Basic. This image shows the virtual buttons that are detected using the segmentation algorithm. The picture on the top left shows the standard image which is being compared with the one in the right. This is done based on the Image Comparison and Enhancement Technique.

START button is selected by the user by blocking it, which is shown in Fig.7. On selecting START button, the bulb is switched ON as shown in Fig. 8.

STOP button is selected by the user by blocking it. On selecting STOP button, the bulb is switched OFF as shown in Fig. 9.

RIGHT button is selected by the user by blocking it. On selecting RIGHT button, the Robot moves right side as shown in Fig 10.

LEFT button is selected by the user by blocking it. On selecting LEFT button, Robot moves left side as shown in Figure 11.

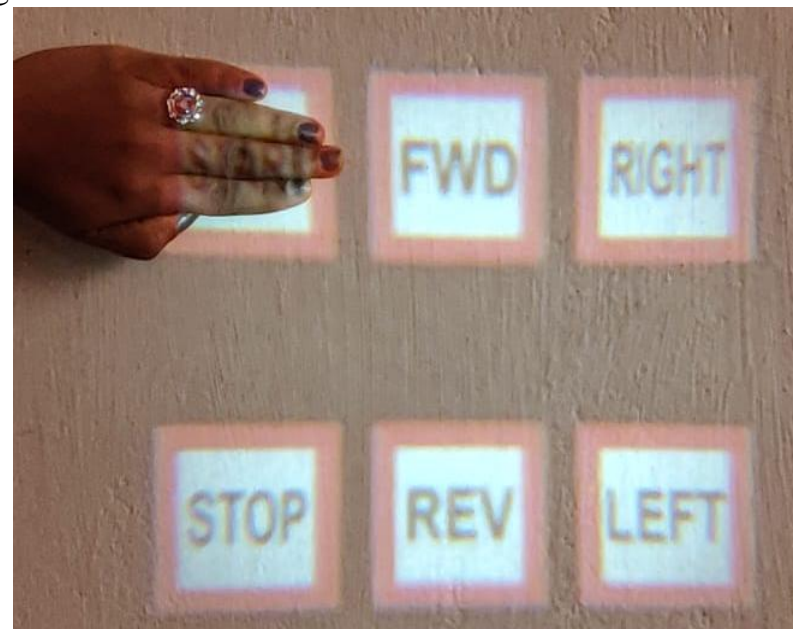

Fig.7. START button selected

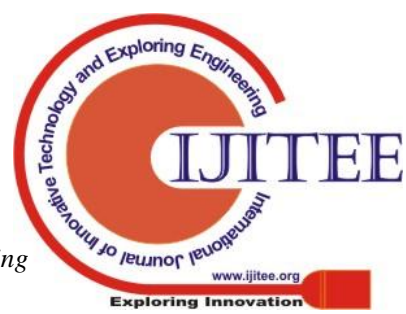




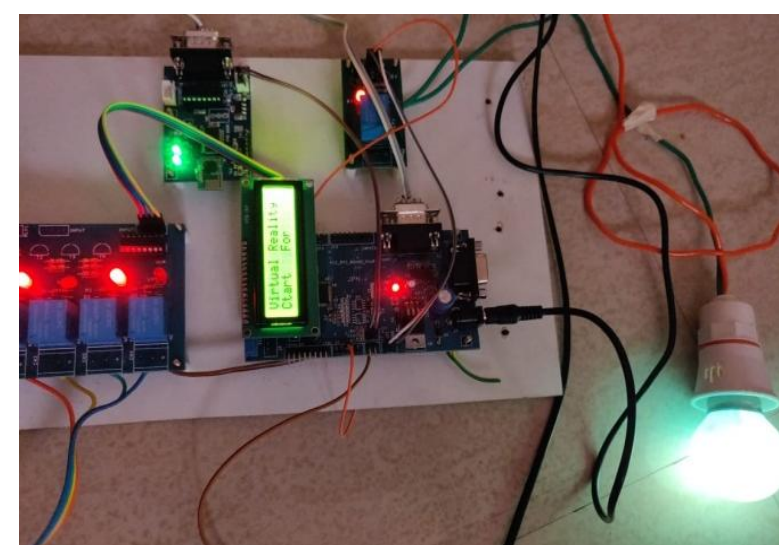

Fig. 8. Bulb turns ON

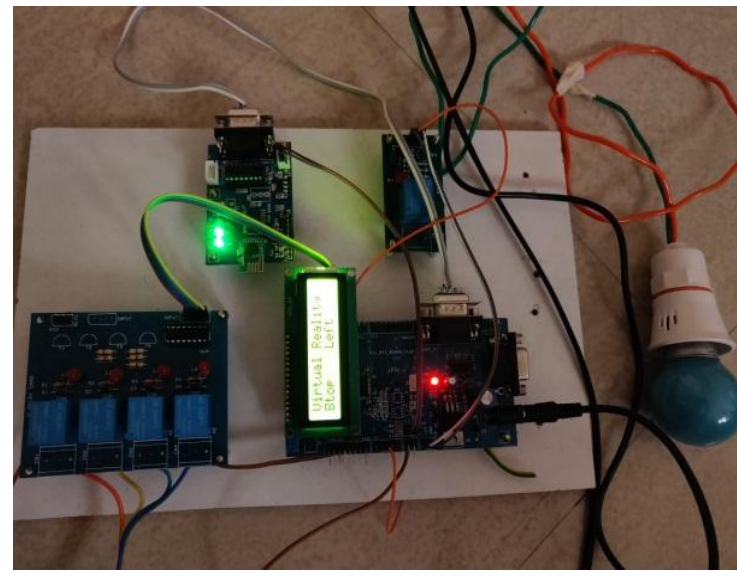

Fig. 9. Bulb turns OFF

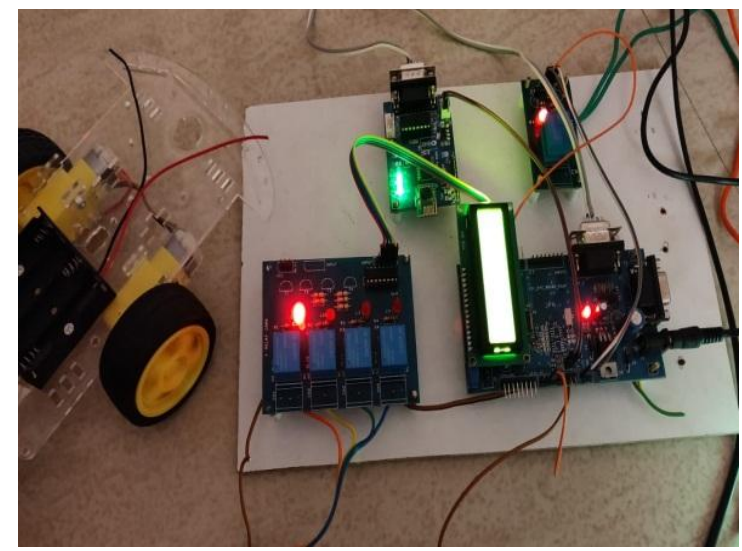

Fig. 10. Robot moves Right

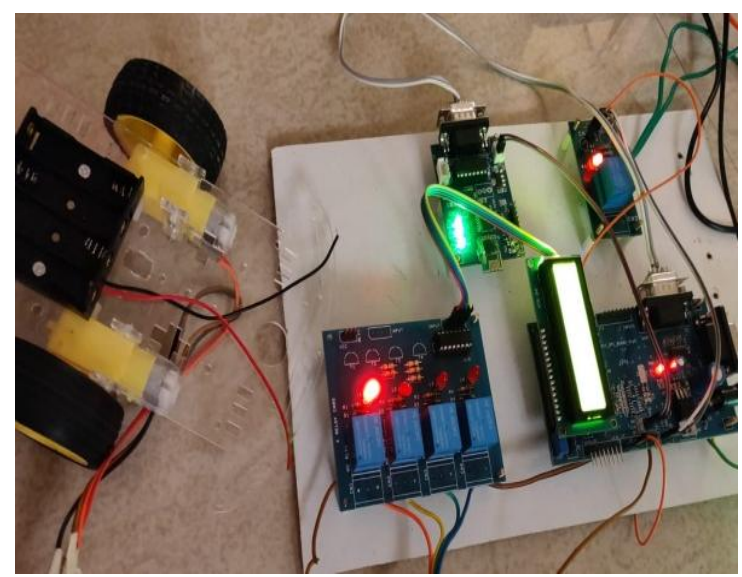

Fig.11. Robot moves Left

\section{CONCLUSION}

Research work has been carried out to develop a new system for controlling the devices which proves to be more suitable in an industrial environment where other modes of control such as using voice commands and usage of electromagnetic radiations are not possible. Detailed design, implementation and evaluation of the completed model has been presented in this paper. Based on the obtained results from practical testing, it is concluded that the proposed virtual-reality based system can alleviate the problems faced in industries where physical buttons can be replaced and in testing environments that cannot utilize radio frequencies to control devices. This model if put into practice, will increase the efficiency and it makes the work easy for even a lay person to control the device(on/off) using a single swipe of hand movement.

Currently, we've created a model which is used to control the industrial devices predominantly. This model can be enhanced further where a 3D camera can be used in the future system for better resolution of image. Because of this, accuracy of the system is increased.Future enhancements can be made by use of the smartphone. We can incorporate this idea such that it can be used as a hand held, portable, easy to carry device. It can be made available as an application using which the virtual buttons can be projected anywhere.

\section{REFERENCES}

1. M.B. Mukesh Krishnan, P.Rajasekar, T.Balachander, "Virtual Reality Implementation in Home Automation" in International Journal of Scientific \& Engineering Research Volume 9, Issue 2, February-2018, pp. 420422 .

2. Audet, S., Okutomi, M., and Tanaka, M.” Augmenting Moving Planar Surfaces Interactively with Video Projection and a Color Camera". IEEE, Virtual Reality (VRW'12),2012, pp.111-112.

3. Fujiwara, T. and Iwatani, Y."'Interactions with a LineFollower: an Interactive Tabletop System with a Markerless Gesture Interface for Robot Control “, Proceedings of the 2011 IEEE International Conference on Robotics and Biomimetics December 7-11, 2011, Phuket, Thailand,pp.2037-2042

4. Kadambari Deherkar, Glen Martin, Nathaneal George, Vinay Maurya "Gesture Controlled Virtual Reality Based Conferencing", International Conference on Smart City and Emerging Technologies (ICSCET 2018),pp.1-4.

5. Professor R.K.Moje, Mr Chandan Kumar Shukla , Miss Renuka D Kolhe , Miss Sukriti Bhat, "Smart Device Control Using Augmented Reality", International Journal Of Innovative Research In Electrical, Electronics, Instrumentation And Control Engineering, Vol. 3, Issue 4, April 2015, pp.77-80

6. Borkowski, S., Letessier, J., and Crowley, J. L. "Spatial Control of Interactive Surfaces in an Augmented Environment". EHCI/DS-VIS Lecture Notes in Computer Science, vol. 3425,2004, pages 228-244.

7. Borkowski, S., Letessier, J., Bérard, F., and Crowley, J.L.(2006). User-Centric Design of a Vision System for Interactive Applications. IEEE Conf. on Computer Vision Systems (ICVS ‘06), pages 9-15.

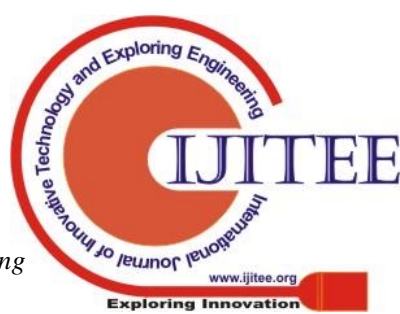


8. Brutzer, S., Höferlin, B., and Heidemann, G. (2011). Evaluation of Background Subtraction Techniques for Video Surveillance. IEEE Conf. on Computer Vision and Pattern Recognition (CVPR '11), pages 1937-1944.

9. Dr.M.B. Mukesh Krishnan , T.Balachander and P.Rajasekar, "Most favorable control routing technique", in International Journal of Engineering and Technology (IJET) p-ISSN : 2319-8613 vol. 8 No. 2 May 2016 , pp.647-652.

10. Hilario, M. N. and Cooper stock, J. R. ," Occlusion Detection for Front-Projected Interactive Displays". 2nd International Conf. on Pervasive Computing and Advances in Pervasive Computing, Austrian Computer ,2004,pp.1-6

11. Kale, A., Kenneth, K., and Jaynes, C.," Epipolar Constrained User Pushbutton Selection in Projected Interfaces". IEEE Conf. on Computer Vision and Pattern Recognition Workshops (CVPRW '04),2004,pp.202-214.

12. Dai, J. and Chung, R. (2012). Making any planar surface into a touch-sensitive display by a mere projector and camera. IEEE Conf. on Computer Vision and Pattern Recognition Workshops (CVPRW '12), pages 35-42. 